\title{
Proceeding Paper \\ Measurement of Line Distribution of Thermal Contact Resistance Using Microscopic Lock-In Thermography ${ }^{\dagger}$
}

\author{
Takuya Ishizaki *, Ai Ueno and Hosei Nagano
}

Citation: Ishizaki, T.; Ueno, A.; Nagano, H. Measurement of Line Distribution of Thermal Contact Resistance Using Microscopic Lock-In Thermography. Eng. Proc. 2021, 8, 18. https://doi.org/10.3390/ engproc2021008018

Academic Editors: Giovanni Ferrarini, Paolo Bison and Gianluca Cadelano

Published: 23 November 2021

Publisher's Note: MDPI stays neutral with regard to jurisdictional claims in published maps and institutional affiliations.

Copyright: (c) 2021 by the authors. Licensee MDPI, Basel, Switzerland. This article is an open access article distributed under the terms and conditions of the Creative Commons Attribution (CC BY) license (https:/ / creativecommons.org/licenses/by/ $4.0 /)$.
Department of Mechanical System Engineering, Nagoya University, Furo-cho, Chikusa-ku, Nagoya 4648603, Aichi, Japan; ueno@mech.nagoya-u.ac.jp (A.U.); nagano@mech.nagoya-u.ac.jp (H.N.)

* Correspondence: ishizaki.takuya@f.mbox.nagoya-u.ac.jp; Tel.: +81-52-789-2739

+ Presented at the 16th International Workshop on Advanced Infrared Technology \& Applications, 26-28 October 2021; Available online: https:/ / aita2021.sciforum.net/.

Abstract: This paper proposes a new thermal contact resistance measurement method using lock-in thermography. Using the lock-in thermography with an infrared microscope, the local temperature behavior in the frequency domain across the contact interface was visualized in microscale. Additionally, a new thermal contact resistance measurement principle was constructed considering the superimposition of the reflected and transmitted temperature wave at the boundary and taking into account the intensity distribution of the heating laser as the gaussian distribution, and the specific geometrical condition of the laminated plate sample. As a result of the experiments, the one-dimensional distribution of the thermal contact resistance was obtained along the contact interface from the analysis of the phase lag.

Keywords: thermal contact resistance measurement; lock-in thermography; periodic heating method; micro scale; interfacial thermal conductance

\section{Introduction}

Thermal contact resistance (TCR) is a phenomenon where heat flow is impeded at the contact interface between two materials. A reduction in TCR is critical for efficiently discharging electronic heat waste, particularly for power semi-conductors. However, TCR reduction is difficult because the TCR occurring mechanism is not fully understood. This is due to the complexity of its physical properties and geometrical and mechanical factors. To understand TCR, we evaluated its factors and measured the local TCR. The steady state method, which is generally used in this case, was used to measure the averaged TCR value across the contact interface of a bulk sample. Additionally, as unsteady methods, the thermo-reflectance [1] method and the $3 \omega$ method [2,3] were studied to measure the TCR of a homogenous thin film sample. However, a method to measure local TCR in the contact interface has not yet been established. For this reason, the authors propose a new measurement method of the local TCR and its distribution, using a periodic heating method with lock-in thermography (LIT). So far, we have developed a theoretical model and measurement apparatus with LIT and an IR microscope. It was demonstrated that by using the proposed method, a local TCR can be visually measured [4]. In this paper, to further develop this method, a new method to measure a line distribution of TCR was reported. At first, a theory of measurement was derived by drawing comparisons between the two heat conduction equations that govern each layer of the laminated and glued sample. A measurement apparatus was also constructed with LIT and an IR microscope. The experiment was conducted on an isotropic graphite sample, then, the local TCR and its distribution was obtained.

\section{Measurement Theory}

The measurement method was carried out using the two layers system, consisting of two thin plates, A and B, that have TCR at their interface, as shown in Figure 1. When a 
point distant from the edge of the A layer surface is heated periodically by a laser focused on a spot, the temperature response is expressed as the following [4]:

$$
T_{j=\mathrm{A}, \mathrm{B}}(r, z, t)=2 \cdot e^{i \omega t} \int_{0}^{\infty}\left[\hat{T}_{j}(k, z, s) \cdot(s-i \omega)\right]_{s=i \omega} \cdot J_{0}(k r) k d k .
$$

where,

$$
\hat{T}_{j}(k, z, s)=\frac{Q_{0} a}{2 \sqrt{\pi}} \mathrm{e}^{\left(-\frac{k^{2} a^{2}}{4}\right)} \frac{1}{s-i \omega}\left\{\mathrm{U}_{j}(k, s) \mathrm{e}^{\sigma_{j} z}+\mathrm{V}_{j}(k, s) \mathrm{e}^{-\sigma_{j} z}\right\} .
$$

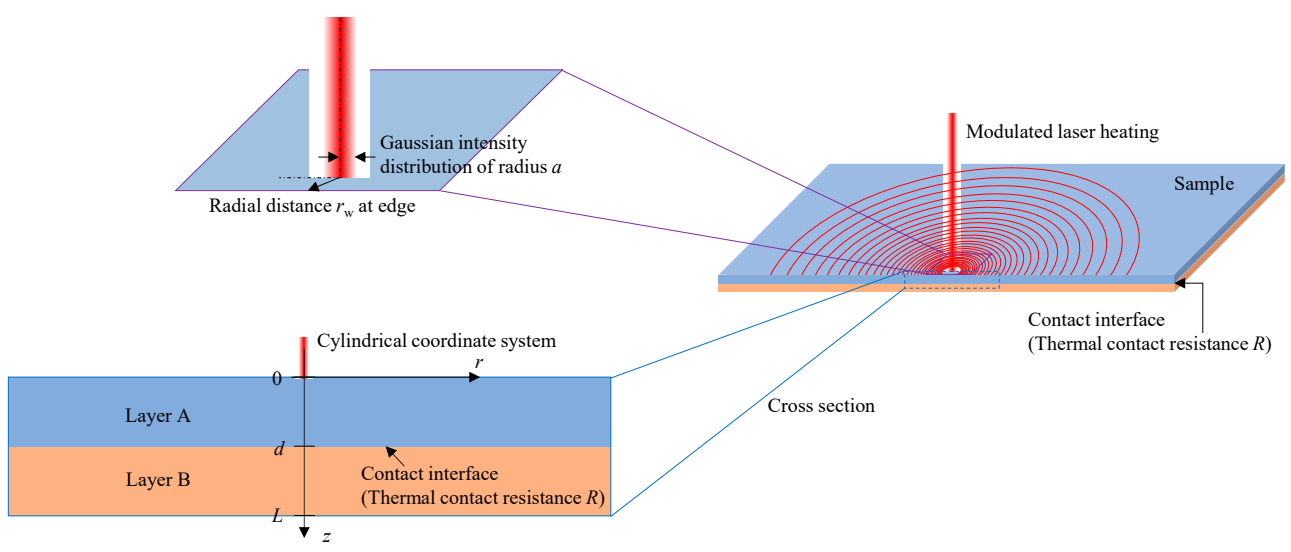

Figure 1. Schematic of TCR measurement theory.

Besides,

$$
\begin{gathered}
\mathrm{U}_{\mathrm{A}}=\frac{1}{\mathrm{G}} \cdot\left\{\lambda_{\mathrm{A}} \sigma_{\mathrm{A}} \cosh \left[\sigma_{\mathrm{B}}(L-d)\right]+\lambda_{\mathrm{B}} \sigma_{\mathrm{B}}\left(\lambda_{\mathrm{A}} \sigma_{\mathrm{A}} R-1\right) \sinh \left[\sigma_{\mathrm{B}}(L-d)\right]\right\} \mathrm{e}^{-\sigma_{\mathrm{A}} d}, \\
\mathrm{~V}_{\mathrm{A}}=\mathrm{U}_{\mathrm{A}}+\frac{Q_{0} a}{2 \sqrt{\pi}} \mathrm{e}^{\left(-\frac{k^{2} a^{2}}{4}\right)} \frac{1}{s-i \omega} \cdot \frac{1}{\lambda_{\mathrm{A}} \sigma_{\mathrm{A}}}, \\
\mathrm{U}_{\mathrm{B}}=\frac{1}{\mathrm{G}} \cdot \lambda_{\mathrm{A}} \sigma_{\mathrm{A}} \mathrm{e}^{-\sigma_{\mathrm{B}} L}, \\
\mathrm{~V}_{\mathrm{B}}=\frac{1}{\mathrm{G}} \cdot \lambda_{\mathrm{A}} \sigma_{\mathrm{A}} \mathrm{e}^{\sigma_{\mathrm{B}} L},
\end{gathered}
$$

$G=2 \lambda_{\mathrm{A}} \sigma_{\mathrm{A}}\left\{\lambda_{\mathrm{B}} \sigma_{\mathrm{B}} \cosh \left[\sigma_{\mathrm{A}} d\right] \sinh \left[\sigma_{\mathrm{B}}(L-d)\right]+\lambda_{\mathrm{A}} \sigma_{\mathrm{A}} \sinh \left[\sigma_{\mathrm{A}} d\right] \cosh \left[\sigma_{\mathrm{B}}(L-d)\right]+\lambda_{\mathrm{A}} \sigma_{\mathrm{A}} \lambda_{\mathrm{B}} \sigma_{\mathrm{B}} \sinh \left[\sigma_{\mathrm{A}} d\right] \sinh \left[\sigma_{\mathrm{B}}(L-d)\right]\right\}$

$T$ is the temperature, the subscribe $\mathrm{A}, \mathrm{B}$ represents layer $\mathrm{A}$ and $\mathrm{B}, r$ is the distance in the radial direction, $z$ is the distance in the thickness direction, $t$ is time, $i$ is imaginary number unit, $\omega$ is angular frequency, "hat" denotes the Hankel transform, $k$ is the Hankel variable, $s$ is the Laplace variable, $J_{0}$ is the zeroth order Bessel function, $Q_{0}$ is the heat source constant, $a$ is the heating radius, $\lambda$ is the thermal conductivity, $d, L$ are the thickness of A layer and whole sample, $R$ is the TCR, and $D$ is the thermal diffusivity, $\sigma_{j}=\sqrt{k^{2}+s / D_{j}}$. The sample surface and the back surface are assumed to be insulated.

Figure 2 shows the theoretical curve of the amplitude and the phase lag in the sample side surface for the different TCR (denoted by $R$ ) with the following applied values: $r=r_{\mathrm{w}}=400 \mathrm{~mm}, f=1 \mathrm{~Hz}, d=0.48 \mathrm{~mm}, L=0.96 \mathrm{~mm}, D_{\mathrm{A}}=D_{\mathrm{B}}=102 \mathrm{~mm}^{2} / \mathrm{s}$, $\lambda_{\mathrm{A}}=\lambda_{\mathrm{B}}=126 \mathrm{~W} /(\mathrm{m} \cdot \mathrm{K}), a=100 \mu \mathrm{m}$, and $R=1.0 \times 10^{-8}-1.0 \times 10^{-5} \mathrm{~m}^{2} \mathrm{~K} / \mathrm{W}$. At the contact interface $(z=d)$, the amplitude and the phase lag jump depending on TCR. Therefore, TCR is analyzed by solving the inverse problem with the measured phase lag by LIT. Since LIT of the IR detector array of $512 \times 640$ can acquire the phase lag distribution of the side surface, we acquired 640 sets of experimental data of the relation between the phase lag and the thickness directional distance. Consequently, TCR distribution from 640 analyses 
is obtained by the analyses along the contact interface with $r$ corresponding to the distance from the sample edge.
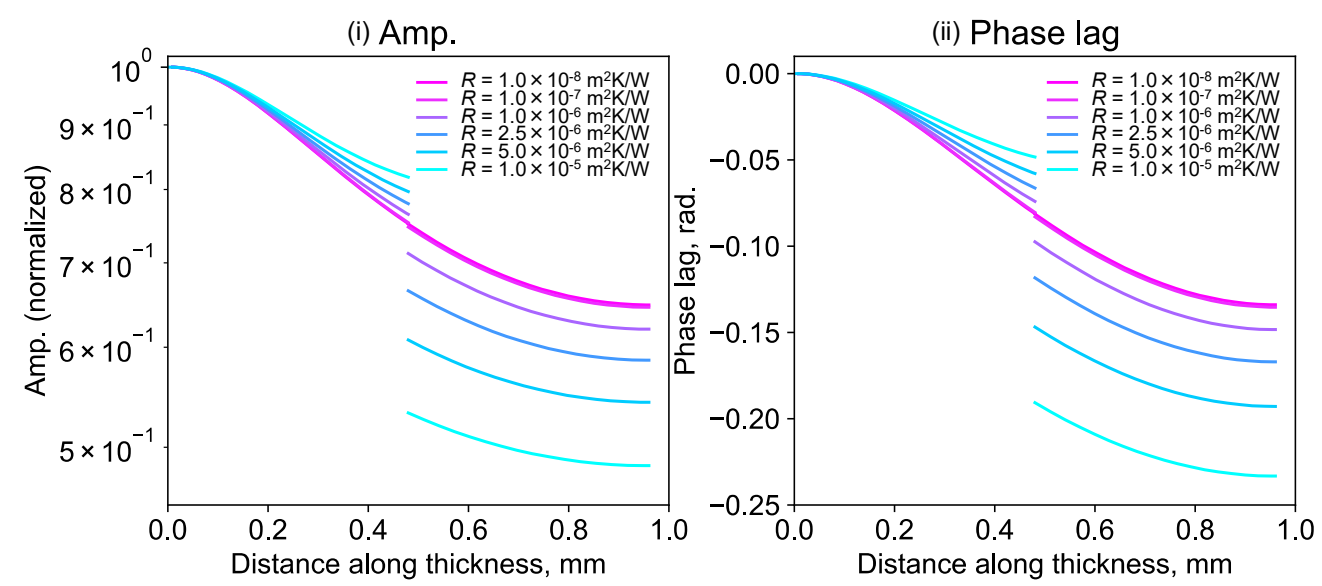

Figure 2. TCR dependence of a theoretical curve of the amplitude ((i) Amp.) and phase lag ((ii) Phase lag) on the sample's side surface.

\section{Measurement Apparatus, Sample and Condition}

The measurement apparatus is shown in Figure 3. It is based on a sample surface spot heating-side surface detecting system in microscale using IR microscope, which has a spatial resolution of $2.5 \mu \mathrm{m}$. A sample was made by bonding and laminating two isotropic graphite IG-110 plates (thickness is $0.480 \mathrm{~mm}$ ). The total thickness of the prepared sample was $0.965 \mathrm{~mm}$. The thermal conductivity and the thermal diffusivity of IG-110 are $126 \mathrm{~W} /(\mathrm{m} \cdot \mathrm{K})$ and $102 \mathrm{~mm}^{2} / \mathrm{s}$ [5]. The measurements were conducted in the conditions of $1.0 \mathrm{~Hz}$. The frame rate of LIT was $99 \mathrm{~Hz}$. The measurement was conducted at room temperature under atmospheric pressure. Heating points were located for the three points of $500 \mu \mathrm{m}$ pitch along the sample edge to assess the validity of the distribution analysis.

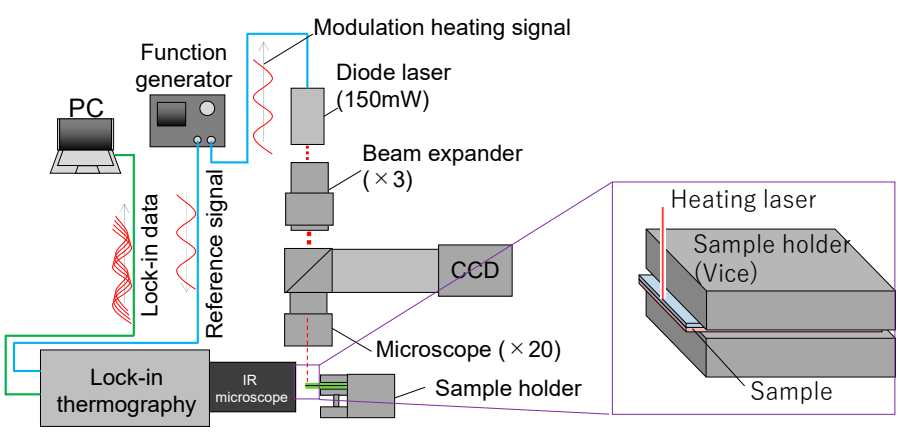

Figure 3. Schematic of TCR measurement apparatus.

\section{Discussion}

Figure 4 shows the TCR measurement result: (a) TCR fitting result for a typical analysis position [4]; (b) IR image of the sample side surface; (c) distribution analysis result. Figure 4a shows the extracted measurement plots and the fitted theoretical curves with error bars showing the standard error rate: ((i) amplitude, (ii) phase lag). The fitting analysis was conducted using the least square method for both the amplitude and phase lag plots. The theoretical curve and experimental plots show a good level of agreement. Thus, the obtained TCR values were $(2.7 \pm 1.5) \times 10^{-6} \mathrm{~m}^{2} \mathrm{~K} / \mathrm{W}$. This result was inferred to provide reasonable values because both were similar to the measurement results of Feng et al. who measured the averaged TCR of a whole contact interface, using the FDTR method with a silicon-silicon contact interface bonded with thermal grease [6]. While the amplitude plots 
have a smaller standard error rate, TCR is more sensitive to amplitude. Therefore, both the amplitude and phase lag, not only the amplitude, were used for the fitting analysis.

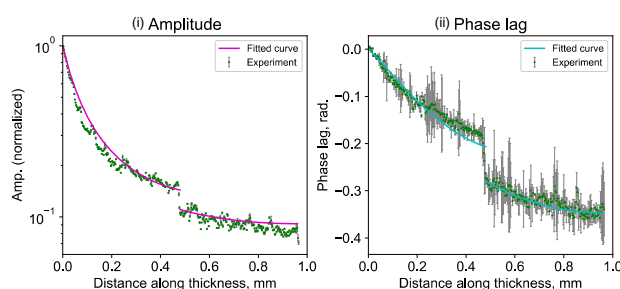

(a)

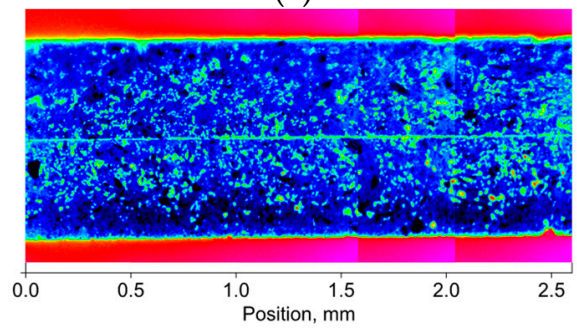

(b)

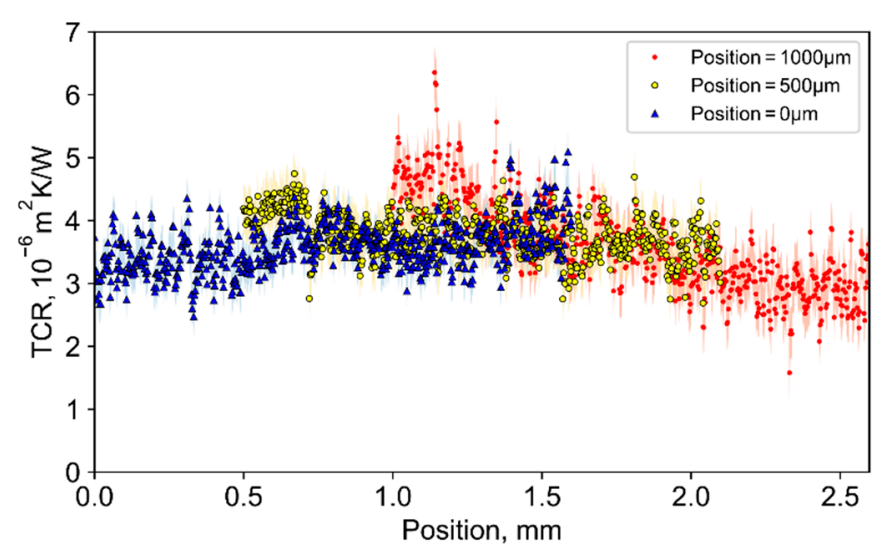

(c)

Figure 4. TCR distribution measurement result: (a) TCR fitting result for a typical analysis position [4]; (b) IR image of sample side surface; (c) distribution analysis result.

Then, the distribution was obtained in the range of $1.6 \mathrm{~mm}$ by one measurement. Figure $4 \mathrm{~b}$ shows the results of the three measurement points at $500 \mu \mathrm{m}$ pitch. The colored shaded area represents the standard error. The validity of the distribution analysis was confirmed by the agreement of the results in the overlapping regions. As a result, it is shown that this measurement method enables the evaluation of the local TCR with a resolution of $2.5 \mu \mathrm{m}$, which matches the spatial resolution of LIT. It was also revealed that there is a difference of about $30 \%$ of TCR distribution in a small area of about $2.6 \mathrm{~mm}$.

\section{Conclusions}

A new measurement method of the local TCR and its distribution was proposed. The measurement theory and apparatus were constructed based on the sample surface spot heating-side surface detecting system in microscale. Then, the validity of the measurement and analysis was confirmed by the experiments with the sample made from isotropic graphite plates. Consequently, the TCR fitting analysis result was $(2.7 \pm 1.5) \times 10^{-6} \mathrm{~m}^{2} \mathrm{~K} / \mathrm{W}$, and it was confirmed to be a reasonable value in comparison of the reference. The values and behavior of the distribution analysis coincided among the results for three measurement points of $500 \mu \mathrm{m}$ pitch. As a result, the distribution analysis revealed that there is a difference of about $30 \%$ of TCR distribution in a small area of about $2.6 \mathrm{~mm}$.

\section{Patents}

A Japanese patent will be certified for the result of this work: JP2019-094934.

Supplementary Materials: The following are available online at https:/ /www.mdpi.com/article/10.3 390/engproc2021008018/s1.

Funding: A part of this research was funded by JST CREST, grant number JPMJCR12I2.

Data Availability Statement: The data presented in this study are available in supplementary material here.

Conflicts of Interest: The authors declare no conflict of interest. 


\section{References}

1. Hopkins, P.E.; Baraket, M.; Barnat, E.V.; Beechem, T.E.; Kearney, S.P.; Duda, J.C.; Robinson, J.T.; Walton, S.G. Manipulating Thermal Conductance at Metal-Graphene Contacts via Chemical Functionalization. Nano Lett. 2012, 12, 590-595. [CrossRef] [PubMed]

2. Moridi, A.; Zhang, L.; Liu, W.; Duvall, S.; Brawley, A.; Jiang, Z.; Yang, S.; Li, C. Characterization of high thermal conductivity thin-film substrate systems and their interface thermal resistance. Surf. Coat. Technol. 2018, 334, 233-242. [CrossRef]

3. Cahill, D.G.; Goodson, K.; Majumdar, A. Thermometry and Thermal Transport in Micro/Nanoscale Solid-State Devices and Structures. J. Heat Transf. 2002, 124, 223-241. [CrossRef]

4. Ishizaki, T.; Igami, T.; Nagano, H. Measurement of Local Thermal Contact Resistance with a Periodic Heating Method Using Microscale Lock-In Thermography. Rev. Sci. Instrum. 2020, 91, 064901. [CrossRef] [PubMed]

5. NMIJ Certified Reference Materials Catalog 2017-2018. Available online: https://www.nmij.jp/service/C/-CRM_Catalog_(JE)_2 017-2018.pdf (accessed on 14 April 2019).

6. Feng, X.; King, C.; Narumanchi, S. General Multilayer Heat Transfer Model for Optical-Based Thermal Characterization Techniques. Int. J. Heat Mass Transf. 2016, 93, 695-706. [CrossRef] 